JOLANTA RACHWALSKA VON REJCHWALD

ORCID: 0000-0003-3159-1942

Université Marie Curie-Skłodowska, Lublin

jolanta.rachwalska@poczta.umcs.lublin.pl

\title{
UN GESTE FANTÔME DU PRÉSIDENT. RECONFIGURATIONS DANS LES RAPPORTS ENTRE L'ÉTAT LAÏC ET LA RELIGION DANS L'ÈRE POST-SÉCULIÈRE
}

Le XXI siècle sera religieux ou ne sera pas ${ }^{1}$.

Dans nos sociétés commémoratives, célébrer les morts sert souvent à faire resurgir l'État et, comme nous le démontrerons, la laïcité de la République. Depuis son élection en 2017, le président Emmanuel Macron a assisté publiquement à deux cérémonies religieuses officielles. La première a eu lieu le 26 juillet 2017, jour du $1^{\mathrm{er}}$ anniversaire de l'assassinat du père Jacques Hamel, tué à l'issue d'une messe qu'il célébrait à Saint-Étienne-du-Rouvray. Emmanuel Macron, en présence de l'archevêque de Rouen, a prononcé un discours dans cette église, lieu de supplice de ce religieux, victime d'un acte terroriste.

La seconde, moins protocolaire, a eu lieu le 9 décembre 2017, en l'église de la Madeleine à Paris et a pris une forme inédite, celle d'un « hommage populaire $»^{2}$ rendu à Johnny Hallyday, icône du rock'n'roll français ${ }^{3}$. À l'intérieur de l'église, au moment de la bénédiction traditionnelle du cercueil, le président Macron a

${ }^{1}$ Formule attribuée à André Malraux : F. Lenoir, « Malraux et le religieux », <https://www. fredericlenoir.com/editos-monde-des-religions/malraux-et-le-religieux/> [consulté le 19.09.2018].

${ }^{2}$ Il n'est pas à confondre avec l'hommage national rendu dernièrement à Simone Veil (le 5 juillet 2017) ou à Jean d'Ormesson (le 8 décembre 2018).

${ }^{3}$ Cf. L. Lavige, Johnny Hallyday : notre icône, Hugo et Compagnie, Paris 2016. 
failli prendre le goupillon et bénir le cercueil, mais il s'est ravisé au dernier moment. Pourtant, comble de l'ironie, son geste fantôme est survenu le jour anniversaire de la loi de séparation de l'Église et de l'État de 1905, étape fondamentale de la laïcisation de la République française.

En sa qualité de chef d'État en exercice, le président est garant de la séparation de l'État et de l'Église. Il incarne même cette séparation, d'où l'importance de son geste dont l'ambiguiité a déchaîné une vague de commentaires : les uns l'ont critiqué ouvertement, voyant dans ce mouvement, pourtant furtif et inabouti, un outrage au principe de laïcité ; d'autres, moins dogmatiques ou plus empathiques, l'ont inscrit dans la logique du rituel de la cérémonie. Le président Macron n'a d'ailleurs pas prononcé d'éloge funèbre - pratique réservée aux hommages nationaux - , mais a simplement pris la parole sur le parvis de l'église et non à l'intérieur, comme il l'avait fait d'ailleurs aussi à Saint-Étienne-du-Rouvray ; cette « délocalisation » de la parole présidentielle démontre la même volonté de ne pas déroger à la loi de 1905 .

Mais qu'en est-il de ces présidents de l'ère post-politique ${ }^{4}$ évoluant dans le contexte post-séculier? Il a suffi d'observer le comportement de François Hollande et Nicolas Sarkozy, deux anciens présidents, devant le cercueil de Hallyday pour se faire une idée de la complexité du problème révélé, par mégarde, par le président Macron. François Hollande, athée déclaré, n'a fait que s'incliner devant le cercueil, gardant la gestuelle sobre ; Nicolas Sarkozy, ouvertement catholique, a fait un signe de croix, ce qu'il faisait déjà alors qu'il était au pouvoir ; et le président Macron, après avoir réprimé son geste, s'est finalement contenté de toucher le cercueil.

On pourrait dire que dans le geste fantôme du président, réprimé illico, « le rationnel l'emporte sur l'irrationnel $»^{5}$. Son geste aussi furtif que symptomatique, taxé par certains journalistes de « moment de flou $»^{6}$, rend ambiguë la posture « jupitérienne » du président Macron et introduit un bougé, un tremblé dans la stature inébranlable de celui qui se conçoit comme le «maître des horloges $»^{7}$. Ce geste

${ }^{4}$ Grosso modo, il s'agit d'un type de politique fondé sur le dépassement du clivage droite/ gauche et sur l'adoption d'une nouvelle forme de gouvernance appelée « centrisme radical » ou stratégie de la troisième voie. Dans la perspective post-politique, il n'y a pas d'alternative à la mondialisation néolibérale ( $c f$. M. Laine, Post-politique, JC Lattès, Paris 2009; Ch. Mouffe, « Macron, stade suprême de la post-politique », <https://www.lemonde.fr/idees/article/2017/06/01/chantal-mouffem-macron-stade-supreme-de-la-post-politique_5136932_3232.html> [consulté le 17.09.2018]).

${ }^{5}$ A. Jacquard, Nouvelle petite philosophie, Stock, Paris 2005, p. 137.

${ }^{6}$ J. Pecnard, «Le moment de flou du président Macron devant le cercueil de Johnny », <https:// www.lexpress.fr/actualite/politique/video-le-moment-de-flou-du-president-macron-devant-le-cercueil-de-johnny_1967684.htm> [consulté le 23.09.2018].

7 L'expression apparaît dans une interview d'Emmanuel Macron par David Pujadas sur France 2, à l'issue du second tour qu'il a remporté sur François Fillon ( $c f$. A. James, « Qui est le maître des horloges invoqué par Emmanuel Macron », <https://www.liberation.fr/politiques/2017/05/17/quiest-le-maitre-des-horloges-invoque-par-emmanuel-macron_1569931> [consulté le 22.09.2018]). 
malencontreux serait-il révélateur d'une tension interne entre deux postures, celle du président de l'État laïc et celle de l'ancien assistant de Paul Ricœur ? ${ }^{8}$ Il est indéniable que la collaboration avec ce grand philosophe a dû fortement influencer Emmanuel Macron dans sa manière de penser la laïcité. Cette imprégnation par la réflexion philosophique se voit confirmée par Philippe Potier, historien des religions et l'un des spécialistes français les plus renommés de la laïcité, qui souligne la singularité de la biographie du président dans un entretien accordé à Libération :

En réponse au philosophe Régis Debray qui l'avait qualifié de «néo-protestant à l'anglo-saxonne », le Président s'est défini à la fois comme l'héritier des jésuites et de la République. Chez lui, la question de la transcendance est importante. L'existence humaine, selon lui, ne peut se réduire à la seule défense d'intérêts séculiers. [...] Même si comme chef de l’État, il ne s'est jamais exprimé publiquement sur son appartenance confessionnelle, il a clairement fait état de son intérêt pour la chose religieuse. Il est vrai qu'il a eu un parcours singulier. À distance des choix de ses parents, il a lui-même demandé le baptême à l'âge de douze ans. Faut-il rappeler qu'une expérience de conversion n'est jamais neutre?

En abandonnant le fortuit et l'anecdotique, nous nous proposons de conférer à ce geste entamé mais jamais abouti la valeur d'un signe révélateur d'un changement dans la manière dont l'État gère ses rapports avec l'Église. Nous ne nous livrerons cependant pas à une étude sémiologique du geste, comme celles qui sont menées en France depuis plus de vingt ans ${ }^{10}$, mais à une analyse du discours politique. Pour la réaliser, nous avons choisi huit discours du président Macron, afin d'y repérer les éléments annonciateurs d'une reconfiguration possible dans les rapports entre l'État et l'Église. La méthode adoptée consistera à analyser ces discours non pas comme des prises de parole isolées, mais dans une lecture séquentielle, régie par la chronologie, ce qui permettra d'y repérer un certain nombre d'invariants interprétables comme les prémisses d'un changement. La thèse que nous nous proposons de défendre dans la présente étude est celle d'un recadrage possible de la manière d'appréhender les rapports entre l'État laïc et la religion. Si nécessaire, mais sans vouloir en faire la règle, nous mettrons les faits et gestes du président en parallèle avec ceux de ses prédécesseurs politiques de la V République. En outre, ce fameux geste de bénédiction manqué nous servira d'embrayeur temporel pour mettre en rapport le passé avec le présent et si

${ }^{8}$ V. de Montclos, « Macron, ses années Ricœur », Le Point, 11 août 2016, pp. 24-27. Dans l'avant-propos à La mémoire, l'histoire, l'oubli (Seuil, Paris 2000), Ricœur insère des remerciements à Macron pour sa relecture studieuse du style et pour l'élaboration de l'outillage critique de cet ouvrage.

${ }^{9}$ B. Sauvaget, « Pour Macron, l'État n'a pas toujours raison sur les religions », Libération, 11 mars 2018, <https://www.liberation.fr/debats/2018/03/11/pour-macron-l-etat-n-a-pas-toujoursraison-sur-les-religions_1635350> [consulté le 20.09.2018].

10 Il s'agit principalement des travaux de Geneviève Calbris (« Geste et motivation », Semiotica 65, 1987, pp. 57 -96; L'expression gestuelle de la pensée d'un homme politique, CNRS, Paris 2003). 
possible avec le futur; il permettra donc de relier un acte fondateur de la sécularisation de la République avec l'hypermodernité définie comme post-séculière.

Cependant, aborder le thème de la religion en France amène à réfléchir sur les deux logiques qui commandent les relations entre la société démocratique et la religion : la logique laïque et celle de la sécularisation ${ }^{11}$. Mais, comme l'explique Marcel Gauchet, qui interroge la place de la religion dans la démocratie, ces deux concepts concurrentiels n'ont aucune valeur explicative : ils sont strictement descriptifs $^{12}$. Sans donc tomber dans les rets des débats définitoires et parfois vains, nous nous concentrons essentiellement sur la perspective post-séculière. Munie de ce préfixe temporel « post » qui marque une position dans le temps, la notion de post-sécularité impose d'emblée une temporalité processuelle, c'est-à-dire qu'elle nous place dans une continuité : « une société post-séculière » doit avoir été séculière, c'est-à-dire avoir été une société « où les liens religieux des citoyens se sont relâchés continûment, même d'une manière drastique depuis la fin de la Seconde Guerre mondiale ${ }^{13}$. Ce processus souligne l'importance du phénomène de la labilité des frontières entre le fait religieux et l'État laïc, ce qui caractérise le post-séculier. Il se peut donc que l'ambiguïté du président et le flou de son attitude se révèlent pertinents pour mieux comprendre l'ère post-séculière en France.

Nous souscrivons à la pensée de Jürgen Habermas qui, dans son article fondateur, nie l'existence d'un lien direct entre sécularisation et modernité. Il constate une " résurgence de la religion », mais qui se manifesterait sous une forme auratique, spectrale, de religion « invisible $»^{14}$; même si elle est amputée officiellement de sa visibilité socio-politique par le culte de la Raison et du Progrès des démocraties modernes, cette religion jugée obsolète perdure et revient subrepticement, par la porte de l'inconscient, comme ré-vélation ${ }^{15}$ d'une part occultée et reniée de la vie sociale. Pourrait-on donc voir dans ce geste présidentiel « une ruse de la raison religieuse ${ }^{16}$ dont parle Jean-Marc Ferry, promoteur de la notion de post-sécularité en France ? La thèse de la résurgence de la religion, qui s'accompagne d'un «changement de conscience » en Europe, constitue le point de départ de notre réflexion qui portera sur « une reconfiguration globale du religieux et du politique $»^{17}$ dans la France du président Macron.

${ }^{11}$ F. Champion, «Entre laïcisation et sécularisation. Des rapports Église-État dans l’Europe communautaire », Le Débat 27, 1993, pp. 46-72.

12 M. Gauchet, La religion dans la démocratie, Gallimard, Paris 2001, p. 18.

13 J. Habermas, «Qu'est-ce qu'une société post-séculière ? », Le Débat 152, 2008, p. 4 (DOI : 10.3917/deba.152.0004).

14 T. Luckmann, "Transformations of Religion and Morality in Modern Europe », Social Compass 3, 2003, pp. 275-285.

15 Pris au sens étymologique de revelare signifiant « dévoiler », de velum, « voile » (J. Dubois, H. Mitterand et al., Dictionnaire étymologique et historique du français, Larousse, Paris 1993).

16 J.-M. Ferry, La Raison et la Foi, Pocket, Paris 2016, p. 93.

17 J.-P. Willaime, «La sécularisation : une exception européenne ? Retour sur un concept et sa discussion en sociologie des religions ", Revue française de sociologie 4, 2006, pp. 755-783. 
Sans vouloir faire entendre que ce président a été l'un des premiers à avoir tenté de modifier la loi de 1905, rappelons qu'elle a fait l'objet de quelques tentatives d'amendements, dont celles de Jean-Pierre Machelon en 2005, de Thierry Tuot en 2013 ou de Manuel Valls en $2015^{18}$. La conception de la laïcité d'Emmanuel Macron est « clairement inspirée de la pensée du philosophe Paul Ricœur ${ }^{19}$ et fondée sur la volonté de faire accéder la question des cultes au débat public. La proposition macronienne

[...] s'inscrit en fait dans une certaine continuité, celle d'une laïcité de dialogue et de reconnaissance. Il est l'héritier d'une conception de la laïcité redessinée sous la Ve République. Grosso modo, elle émerge lorsque l'État rompt progressivement avec le principe de séparation posé par la loi de 1905. À partir de ce moment-là, l'État associe davantage les cultes (comme les autres forces de la société civile) à sa réflexion et à son action. Avant cela, les politiques publiques relevaient de la seule rationalité de l'État ${ }^{20}$.

Le ressenti de ce changement devient peut-être plus frappant du fait que son prédécesseur, François Hollande, séparait la démocratie des religions, ce dont il a donné un témoignage marquant dans son discours du Bourget (21.01.2012) en déclarant que «la démocratie était plus forte que les croyances et les religions $»^{21}$. En général, l'idée de Macron consiste, d'une part, à rejeter la laïcité de combat, c'est-à-dire à ne pas ériger la laïcité en religion d'État, en " religion civile qui viendrait se substituer aux religions historiques »; de l'autre, à ne pas verser dans une laïcité d'abstention. À travers l'analyse des discours présidentiels, nous démontrerons les grands axes de sa vision de la laïcité, qui est fondée sur une ouverture des agoras politique et publique aux religions : « Le Président considère que la religion doit avoir la possibilité d'intervenir dans le jeu de la délibération collective et participer à la configuration des décisions publiques $»^{22}$.

Comme en témoigne l'observation attentive des rapports entre la République française et la religion, il apparaît que malgré la loi de la laïcité, c'est au cœur de la démocratie même qu'a survécu un principe sacré. Pour le prouver, il suffit de penser à une tradition qui remonte au XVII ${ }^{\mathrm{e}}$ siècle et qui pérennise les liens étroits entre la religion et l'État en France. Il s'agit du titre de « premier et unique chanoine honoraire » de la basilique Saint-Jean-de-Latran à Rome. Ce titre honorifique revient de droit aux chefs d'État français en exercice depuis Henri IV (depuis 1604), parce qu'ils sont considérés comme successeurs des rois de France.

$18 C f$. C. Kintzler, «"Radicalisation" de la laïcité : les propos ambigus du président Macron », Le Causeur, 2 janvier 2018, <https://www.causeur.fr/emmanuel-macron-laicite-radicalisation-societe $>$ [consulté le 21.09.2018].

${ }^{19}$ B. Sauvaget, op. cit.

${ }^{20}$ Ibidem.

${ }^{21}$ Discours de François Hollande au Bourget (<https://www.nouvelobs.com/election-presidentielle-2012/sources-brutes/20120122.OBS9488/1-integralite-du-discours-de-francois-hollandeau-bourget.html $>$ [consulté le 20.09.2018]).

22 B. Sauvaget, op. cit. 
Macron a accepté, comme la plupart de ses prédécesseurs, ce titre de chanoine ${ }^{23}$, mais il s'est abstenu de prononcer le moindre discours pour ne pas envenimer la critique de la part de l'opposition, qui l'attaquait à cause de sa participation, le 9 avril 2018, à la conférence des évêques de France. Pourtant, avant d'avoir reçu ce titre, il s'est entretenu le 26 mai 2018 avec le Pape François, lors d'une audience privée qui a duré 57 min (contre 52 accordées à Obama $)^{24}$.

Avant de revenir sur la présidence de Macron, rappelons quelques faits qui ont marqué les rapports État-Église en France. Sous la Ve République, seuls Pompidou, Mitterrand et Hollande, ayant accepté le titre par tradition, ne se sont pas déplacés en personne à Rome pour être institués chanoines. Par contre, le discours du président Nicolas Sarkozy, qui s'y est rendu en 2007, a provoqué un grand tollé médiatico-politique à cause de son apologie de la foi, censée compromettre, selon la gauche, le principe de laïcité. Dans un entretien exclusif accordé au Figaro Magazine, questionné sur son discours de Latran dans lequel il avait mis en avant les racines chrétiennes de la France, Sarkozy a dit ne rien regretter :

Mais enfin, la France a des racines chrétiennes, et même judéo-chrétiennes, c'est une réalité historique qu'il serait absurde de nier. [...] La France est née de la rencontre entre la volonté des rois et celle de l'Église. Jeanne d'Arc, dont on vient de célébrer le $600^{\mathrm{e}}$ anniversaire de la naissance, est au carrefour de cette double volonté. Dire cela ne signifie pas qu'on n'appartient à une Église, ni qu'on adhère moins aux valeurs de la République ou au principe de laïcité. N'amputons pas la France d'une partie de son histoire ${ }^{25}$.

Nous proposons donc de soumettre à l'étude huit discours officiels d'Emmanuel Macron qui nous serviront de corpus d'analyse. Nous les avons choisis en fonction de leur pertinence thématique et de la résonance politique qu'ils ont eue dans l'opinion publique en France. Leur analyse nous permettra de repérer les idées phares qui reconfigurent la place de la religion au sein de l'État laïc. Leurs transcriptions officielles sont accessibles sur le site web du Palais de l'Élysée, et leur liste complète est présentée ci-dessous, dans l'ordre chronologique, qui sera aussi celui de notre argumentation :

Discours devant le Conseil français du culte musulman, 21.06.2017

Discours à Saint-Étienne du Rouvray, 26.07.2017

Discours à l'occasion du 500 anniversaire de la Réforme protestante, 22.09.2017

${ }^{23}$ A.-A. Durand, S. Laurent, « Pourquoi le président français devient-il le chanoine de Latran ? », Le Monde, 26 juin 2018, <https://www.lemonde.fr/les-decodeurs/article/2018/06/26/pourquoi-le-president-francais-devient-il-chanoine-de-latran_5321396_4355770.html > [consulté le 11.02.2019].

${ }^{24}$ G. Ottenheimer, « Emmanuel Macron rêve de réconcilier République et chrétienté », Challenges, 26 juin 2018, <https://www.challenges.fr/politique/emmanuel-macron-reve-de-reconcilier-republique-et-chretiente_596986> [consulté le 10.02.2019].

25 A. Brézet, C. Meeus, « Nicolas Sarkozy : mes valeurs pour la France », Le Figaro magazine, 11 février 2012, p. 38. 
Allocution avant la messe funéraire de Johnny Hallyday, 9.12.2017

Discours aux représentants des cultes, 21.12.2017

Discours des vœux aux autorités religieuses, 6.01.2018

Discours devant le Conseil représentatif des institutions juives, 9.03.2018

Discours à la Conférence des évêques de France, 9.04.2018

\section{DISCOURS DEVANT LE CONSEIL FRANÇAIS DU CULTE MUSULMAN}

Le premier dans l'ordre chronologique est un discours prononcé le 21 juin 2017 devant le Conseil français du culte musulman $(\mathrm{CFCM})^{26}$. Présenté dans le contexte des attentats terroristes, c'est autour du constat d' « une déchirure profonde entre les Français de toutes les croyances et les Français de confession musulmane » que se concentre la parole présidentielle. Prenant acte de l'existence de cette " déchirure profonde », Emmanuel Macron réaffirme sa conviction que « les Français de tous horizons, de toutes convictions, tous citoyens au sein d'une République » jouissent de la liberté garantie par la laïcité. En même temps, allant dans le sens de la laïcité d'ouverture, il formule une sorte de mise en garde (« il faut être vigilant ») contre la tentative d'un repli identitaire autour de sa propre religion, ce qui serait incompatible avec l'esprit laïc de la République. Qui plus est, toujours dans le sens de l'ouverture, il tient un discours de conciliation et d'inclusion en réaffirmant le principe de laïcité suivant lequel «personne ne peut faire croire que la France rejetterait sa composante de foi musulmane ».

\section{DISCOURS À SAINT-ÉTIENNE DU ROUVRAY}

Le 26 juillet 2017, le président se rend à Saint-Étienne du Rouvray pour commémorer, en compagnie de l'archevêque de Rouen Dominique Lebrun, le $1^{\mathrm{er}}$ anniversaire de l'assassinat du père Jacques Hamel (SER) ${ }^{27}$. Dans son discours, le président rebondit sur les événements tragiques survenus « en ces temps troublés » au cours desquels le père Hamel a été tué dans l'église où il officiait. Un an après cette attaque terroriste, le chef de l'État souligne son attachement à des valeurs républicaines non négociables : « Ces deux meurtriers ont rappelé à tous les Français que la République n'est pas le règne du relativisme. Au cœur de nos lois et de nos codes forgés par l'Histoire, il est une part qui ne se négocie pas. Il est une part sur laquelle on ne porte pas la main. Une part, j'ose le mot, sacrée ». Le risque sémantique qu'il

${ }^{26}$ Texte intégral du discours : $<$ https://www.elysee.fr/emmanuel-macron/2017/06/21/discours-dupresident-de-la-republique-au-diner-du-conseil-francais-du-culte-musulman $>$ [consulté le 20.09.2018].

${ }^{27}$ Texte intégral du discours : < https://www.elysee.fr/emmanuel-macron/2018/01/09/discours-demmanuel-macron-en-hommage-au-pere-hamel-a-saint-etienne-du-rouvray-2> [consulté le 20.09.2018]. 
prend rajoute à l'ambiguïté de sa position, mais il évoquera cette dimension sacrée encore dans d'autres discours, car elle semble incarner sa vision de la laïcité : « La République garantit la liberté de croire, comme de ne pas croire. La République n'a pas à combattre une religion, ni à vouloir se substituer à elle. Elle œuvre à ce que chacun puisse croire ou pas dans l'intensité et l'intimité de sa foi ».

\section{DISCOURS À L'OCCASION DU 500 ANNIVERSAIRE DE LA RÉFORME PROTESTANTE}

Deux mois plus tard, le 22 septembre 2017, le président Macron prononce un discours à l'occasion du $500^{\mathrm{e}}$ anniversaire de la Réforme protestante (RP) ${ }^{28}$. Il en profite pour revenir sur la question de la laïcité, « dont trop souvent on méconnaît la nature » (RP). Il explique que « la laïcité, ce n'est pas une religion d'État, c'est une exigence politique et philosophique, ce n'est pas la négation des religions, c'est la capacité à les faire coexister dans un dialogue permanent ». Le président intervient aussi sur deux manières d'appréhender la religion au sein de l'État laïc, à savoir comme un fait culturel et un objet de foi : « Nous pouvons adopter à l'égard des religions une vision entièrement culturelle et considérer qu'une religion se définit par des rites, des usages sociaux [...] ». Cette acception s'oppose à la religion associée à la foi que professent les protestants. Selon Macron, la foi protestante ne sert pas au repli identitaire de cette communauté de confession, mais « se mêle à l'Europe, c'est une histoire européenne qui va essaimant à travers le monde ». Ainsi, il associe l'idée de l'ouverture à la République, mais il évoque aussi l'idée du resserrement de l'Europe grâce au lien du « projet humaniste ».

\section{ALLOCUTION AVANT LA MESSE FUNÉRAIRE DE JOHNNY HALLYDAY}

Le 9 décembre 2017, Emmanuel Macron participe à l'hommage populaire à Johnny Hallyday et prononce un bref discours funéraire sur le parvis de l'église de la Madeleine $(\mathrm{JH})^{29}$. Tout en faisant l'éloge du talent du chanteur, il insiste sur le fait que ce grand rassemblement populaire autour de Johnny peut servir aussi à resserrer des liens d'unité et de solidarité dans la nation. Son intention de récupérer l'événement au profit de l'idée de l'intégration nationale est perceptible dans les formules qui relèvent de la rhétorique de l'insistance : « nous sommes ensemble », « nous sommes une nation », « nous sommes un peuple uni » : « Il

${ }^{28}$ Texte intégral du discours : <https://www.elysee.fr/emmanuel-macron/2017/09/25/discours-dupresident-de-la-republique-emmanuel-macron-a-loccasion-du-500e-anniversaire-de-la-reforme-protestante-hotel-de-ville-paris $>$ [consulté le 20.09.2018].

${ }^{29}$ Texte intégral de l'allocution : < https://www.elysee.fr/emmanuel-macron/2017/12/09/hommage-populaire-a-johnny-hallyday> [consulté le 20.09.2018]. 
[Johnny] a traversé le temps, les époques, les générations et tout ce qui divise la société. Et c'est pour cela que nous sommes ensemble aujourd'hui, c'est aussi pour cela que je m'exprime devant vous. Parce que nous sommes une nation qui dit sa reconnaissance. Parce que nous sommes un peuple uni autour d'un de ses fils prodigues $»$.

\section{DISCOURS AUX REPRÉSENTANTS DES CULTES}

Le 21 décembre 2017, pour désamorcer un climat de tension autour de la question de la laïcité, le président Macron reçoit à l'Élysée les représentants des six grandes religions de France : catholique, protestante, orthodoxe, musulmane, juive et bouddhiste. Aucun autre président avant lui n'avait rencontré les représentants de tous les grands cultes. Selon les propos recueillis après cette rencontre par les journalistes ${ }^{30}$, le président a annoncé son projet de grand discours sur la laïcité pour le « début 2018 ». En outre, il a affirmé devant ses hôtes que « selon la philosophie de la loi 1905, c'est la République qui est laïque et non la société » et qu'il faut rester vigilant par rapport à la « radicalisation de la laïcité ». Ces deux formules, qui ont fait couler beaucoup d'encre en France, semblent subsumer sa conception de la laïcité, même si elles sont très ambiguës ${ }^{31}$, tout comme son geste fantôme. Même s'il serait prématuré d'affirmer un réel changement dans les rapports Église-État, il est déjà possible de parler d'un évident changement dans le discours : il est perceptible dans ces deux formules du président.

\section{DISCOURS DES VEUX AUX AUTORITÉS RELIGIEUSES}

Le 4 janvier 2018, Emmanuel Macron réunit à nouveau les représentants des six principales religions pour une cérémonie de vœux (DV) $)^{32}$ qui s'inscrit dans une tradition établie en 1947 par Vincent Auriol, promoteur d'une " laïcité de combat $»$. Le président souligne un changement visible dans la nature de cette cérémonie qui, d'une audience protocolaire, se transforme en une " cérémonie commune restituant enfin l'image d'un paysage de foi divers que l'État considère sans défaveur ni parti pris », parce que « l'État n'est partie prenante d'aucun culte mais garantit à chacun son libre exercice dans le strict respect des lois de la République, comme il garantit aussi la liberté de ne pas croire dans les mêmes

${ }^{30}$ C. Chambraud et S. de Royer, « Climat de confiance entre Macron et les représentants religieux », Le Monde, 22 décembre 2017, <https://www.lemonde.fr/religions/article/2017/12/22/climat-de-confianceentre-macron-et-les-representants-religieux_5233273_1653130.html $>$ [consulté le 20.09.2018].

${ }^{31}$ C. Kintzler, op. cit.

32 Texte intégral du discours : <https://www.elysee.fr/emmanuel-macron/2018/01/04/transcriptiondu-discours-des-v-ux-du-president-de-la-republique-aux-autorites-religieuses $>$ [consulté le 20.09.2018]. 
conditions de neutralité ». Il a complété cette définition de la laïcité en expliquant que « [c]e sécularisme à la française, qui parfois surprend nos voisins, est un ciment puissant dans notre pays qu'ont déchiré tant de guerres de religions, où la religion est inscrite dans l'héritage intellectuel, culturel, social ». Dans la suite, il évoque la nécessité de "quelque pédagogie », car certains font un amalgame entre la neutralité et l'absence de l'État. Il explique que cette neutralité de l'État laïc sécrète un vide que viendrait occuper la laïcité érigée en religion d'État : «Et je ne souhaite pas qu'une religion d'État soit substituée de cette manière aux religions. Mais pas davantage la religion ne saurait colorer la vie politique de la nation ». Macron rejette cette idée avec fermeté, se mettant dans le sillage de Raymond Aron qui nous interpelle : « une doctrine sans Dieu mériterait-elle d'être appelée religion ? $»^{33}$. Cependant, le président revient à plusieurs reprises sur « la persistance des religions voire la résurgence sous des formes variées de la préoccupation spirituelle », car " l'individu nourrit toujours une interrogation existentielle que le vide inquiète, insécurise ».

Il se montre lucide quant à " l'inévitable friction entre l'ordre religieux et l'ordre politique », mais affirme que « cette distinction des Ordres est un acquis précieux [...] pour la République [...] ». Il ajoute toutefois que, malgré l'appartenance à différents Ordres, " il est naturel que le président de la République s'entretienne régulièrement avec vous parce que vous participez de la vie de la nation ». Macron révèle ici sa conception d'une laïcité ouverte qui se veut conciliatrice, car « la foi religieuse qui relève de l'intime ne disqualifie pas pour être citoyen, il serait fou de penser qu'en une même personne les deux ne dialoguent pas constamment ». Il ajoute que " La République ne demande à personne d'oublier sa foi, mais pour faire nation, il faut également savoir dépasser ses différences en les mettant au service de la communauté de citoyens [...]». Le président, invitant tous les représentants à un travail commun sur le dossier bioéthique, rappelle qu' « [i]l est impensable de penser trouver le bien commun de notre société sans prendre pleinement en considération les religions ». Ce qui interpelle dans ce passage, c'est un changement de registre, car le terme « le bien commun », propre au langage religieux, a été employé au lieu de l' " intérêt général », terme républicain $^{34}$, ce qui dénote une friction du religieux et du politique.

À la fin, il insiste sur la singularité des liens entre l'État et la religion :

Et je souhaite qu'ensemble nous puissions faire de la France, ce lieu où nous ne cachons rien des religions et du fait religieux, où le pluralisme des religions est pleinement reconnu et peut s'épanouir et où nous savons dans le même temps traiter de cette grande fracture du monde. [...] Nous savons tous ce que notre nation doit au cours de son histoire aux multiples apports religieux et philosophiques et le dire n'est pas renoncer à notre pacte laïque mais plutôt, reconnaître cette aspiration qui continue d'animer nombre de nos contemporains à une forme de transcendance que Jaurès décrivait

\footnotetext{
${ }^{33}$ R. Aron, L'Opium des intellectuels, Gallimard, Paris 1968, p. 360.

${ }^{34}$ B. Sauvaget, op. cit.
} 
en ces termes. Il serait mortel de comprimer les aspirations religieuses de l'âme humaine, dès lors qu'il aura dans l'ordre social réalisé la justice, l'homme s'apercevra qu'il lui reste un vide immense à remplir.

C'est avec un certain étonnement qu'on peut constater que le président de l'État laïc, après avoir évoqué auparavant « une part sacrée », parle cette fois d'un vide métaphysique qui s'ouvre dans tout être humain, fatigué par le « tumulte du monde », et le conduit vers la transcendance pour « être comblé ».

\section{DISCOURS DEVANT LE CONSEIL REPRÉSENTATIF DES INSTITUTIONS JUIVES}

Le 7 mars 2018, Emmanuel Macron prononce un discours devant le conseil représentatif des institutions juives de France (CRIF) ${ }^{35}$ dans lequel il explique sa manière de comprendre la laïcité. Selon lui, elle se ramène à bien vivre avec l'autre :

[...] mais la laïcité, pardon mais c'est la réalité, je crois à la laïcité de 1905, mais la laïcité de 1905, elle a toujours été la sœur du bon sens républicain. Et le bon sens républicain, c'est que chacun soit là et c'est qu'un petit enfant de la République qu'il soit juif, qu'il soit musulman, qu'il soit protestant, qu'il soit catholique, il doit être dans l'école de la République au maximum parce que c'est là qu'il recevra l'enseignement de la République et parce que c'est là qu'il apprendra à vivre avec l'autre. Et c'est parce que c'est ça le cœur de la laïcité.

\section{DISCOURS À LA CONFÉRENCE DES ÉVÊQUES DE FRANCE}

Un mois plus tard, le 9 avril 2018, le président participe à la conférence des évêques de France au Collège des Bernardins et prononce un discours ${ }^{36}$, très controversé, qui lui a valu une critique massive de toutes parts. Il affirme que « la sécularisation ne saurait éliminer la longue tradition chrétienne ». Là où Nicolas Sarkozy parlait d'un lien historique, Emmanuel Macron parle de spiritualité et d'absolu. Il développe sa compréhension de la laïcité qui « n'a pas pour fonction de nier le spirituel au nom du temporel, ni de déraciner de nos sociétés la part sacrée qui nourrit tant de nos concitoyens ». Adoptant dans son discours un ton alarmiste, il évoque « ce moment de grande fragilité sociale, quand l'étoffe de la nation risque de se déchirer [...]», et il brosse à grands traits l'histoire des dernières années, marquée par l'exclusion des catholiques des décisions politiques. Il

35 Texte intégral du discours : < https://www.elysee.fr/emmanuel-macron/2018/03/07/conseil-representatif-des-institutions-juives-de-france $>$ [consulté le 20.09.2018].

${ }^{36}$ Texte intégral du discours : <https://www.elysee.fr/emmanuel-macron/2018/04/09/discoursdu-president-de-la-republique-emmanuel-macron-a-la-conference-des-eveques-de-france-au-collegedes-bernardins $>$ [consulté le 20.09.2018]. 
insiste sur le fait que l'État et l'Église « appartiennent à deux ordres institutionnels différents », et que « le temps de l'Église n'est pas celui du monde et pas celui de la politique telle qu'elle va ». L'Église, cette " institution aussi fragile que du verre », doit se transformer, ajoute-t-il, car c'est uniquement avec l'Église, mais celle " moderne ", « en dialogue ", « adaptée à la sécularisation », avec une Église qui n'est pas « intempestive », sachant « accepter le contretemps avec la politique », que l'État peut collaborer. C'est à une telle Église remodelée qu'il s'adresse pour lui demander de " s'engager politiquement dans [le] débat national ». Ce terme d'" engagement » est de poids dans ce contexte, car le président — en esquissant le panorama des dernières décennies - a appelé cette ère postmoderne, «l'ère du grand doute ». Étant donné le « contexte de décrue des solidarités », le plus grand danger pour la société, selon le diagnostic qu'il livre, « c'est l'anomie, l'atonie, l'assoupissement ». Or, la religion, d'après lui, pourrait servir d'instrument pour recentrer la cohésion, pour recoudre la fracture. Ce qui interpelle dans ce discours, c'est qu'il renonce au motif habituel des racines, employé par exemple par Sarkozy, et parle de « la sève catholique [qui] doit contribuer à faire vivre notre nation ». Or, la « sève » de la religion chrétienne devrait avoir pour fonction de revigorer cette nation. Pour expliquer son idée, il fait appel au potentiel spirituel de la religion, qui répond au besoin de transcendance, car « les citoyens ont soif d'absolu » et « l'action politique a besoin des principaux fondements : y compris des croyances spirituelles ». Qui plus est, « [l'Église] dans l'horizon séculier instille la question intranquille du salut », elle est « source d'incertitude qui parcourt toute vie, et qui fait du dialogue, de la question, de la quête, le cœur même du sens ${ }^{37}$. Pour cette raison, Macron propose de « réparer le lien qui s'est abîmé entre l'Église et l'État ». Cette phrase a déclenché en France une véritable tempête médiatique parmi « les hygiénistes de la laïcité » ${ }^{38}$ et a aiguillonné la presse étrangère ${ }^{39}$.

Selon l'Union des familles laïques, ce discours comporte de « multiples dérives, voire des entorses à la laïcité, qui tendent toutes vers une rupture avec le principe de séparation $\gg{ }^{40}$. Effectivement, ce discours, dans lequel le président, censé préserver le principe de laïcité, parle du « salut », du besoin d'absolu et

37 Cf. J. Derrida, G. Vattimo, La religion : Séminaire de Capri, Seuil, Paris 1996, pp. 52-57.

38 A. de Soupa, «La pensée de Macron sur la place des religions dans la société », <https:// mission-universelle.catholique.fr/se-former/articles-et-revues/297836-pensee-de-macron-place-religions-societe> [consulté le 20.09.2018].

39 «Vu de l'étranger. Macron devant les évêques : quelle laïcité pour la France, <https://www. courrierinternational.com/article/vu-de-letranger-macron-devant-les-eveques-quelle-laicite-pourla-france> [consulté le 21.09.2018].

${ }^{40}$ B. Couturier, « Macron en fait-il trop pour les cathos ? », <http://www.lepoint.fr/debats/bricecouturier-macron-en-fait-il-trop-pour-les-cathos-27-06-2018-2231164_2.php> [consulté le 20.09.2018]. 
de la nécessité de réparer le lien entre l'État et l’Église, « électrise la droite et la gauche », laissant sidérée toute la scène politique qui oscille entre " un profond embarras à droite et une franche indignation à gauche $»^{41}$.

\section{CONCLUSION}

Dans son ouvrage au titre paradoxal, Une brève histoire de l'avenir, Jacques Attali écrit, en se projetant dans le futur inconnu des années trente du XXI siècle, que « les valeurs religieuses retrouveront une visibilité politique $»^{42}$. Les discours susmentionnés, qui forment une sorte de théologie politique du président Macron, démontrent la probabilité de cette thèse. Même si Emmanuel Macron s'est abstenu de faire un grand discours sur la laïcité, ces huit interventions, à en juger par la violence des réactions suscitées dans la sphère politique, marquent un tournant dans la manière de gérer la laïcité et contribuent à dessiner les contours des « nouveaux imaginaires sociaux $»^{43}$ en France.

Dans le descriptif de la société qui se dégage des lignes de ses discours, Macron part de l'image de la « déchirure » du tissu social, causée par la "radicalisation de la laïcité », pour aboutir au constat de la nécessité de le "réparer ». Ce qui ressort de sa parole, c'est que le lien, décliné en plusieurs variantes thématiques, serait au cœur de sa préoccupation. Il est donc possible d'en déduire, grâce à la récurrence de cette image, que l'idée du lien semble prévaloir dans sa pensée sur la République (laïque), et que la nation a besoin d'un lien nouveau capable de recoudre le tissu social abîmé, bref, qu'elle a besoin d'un nouveau re-ligare. Ce mot, signifiant « qui noue des liens $»^{44}$, demeure une des deux acceptions étymologiques du terme « religion ». Or, si nous voulions pousser notre raisonnement jusqu'au bout, il en résulterait que cette reconfiguration des rapports État-Religion semble basée sur le renouveau du lien. La nostalgie du lien, perceptible dans le retour de l'image de la « fracture », de la « déchirure », la nécessité d'un nouveau re-ligare républicain et spirituel condenseraient-elles donc, en ces « temps troublés » de l'hypermodernité, le sens d'une nouvelle post-laïcité ?

In fine, pourrait-on entrevoir dans le flou du geste présidentiel les prodromes d'un nouveau rôle que l'État laïc pourrait jouer, les contours d'un nouveau pacte social ? Ou, tout simplement, une amorce de la troisième voie, conciliatrice, dont

${ }^{41}$ L. Delaporte, P. Graulle, «Adresse aux catholiques : Macron électrise droite et gauche », Médiapart, 10 avril 2018, <https://www.mediapart.fr/journal/france/100418/adresse-aux-catholiques -macron-electrise-droite-et-gauche?onglet=full $>$ [consulté le 19.09.2018].

42 J. Attali, Une brève histoire de l'avenir, Fayard, Paris 2006, p. 237.

${ }^{43}$ Le terme est de Ch. Taylor, Modern Social Imaginaires, Duke University Press, Durham (NC) 2004.

44 J. Derrida, G. Vattimo, op. cit., pp. 50-55. 
parle Habermas ${ }^{45}$, entre le fondamentalisme religieux et une laïcité dogmatique, afin que les gens du « cube » et de la « cathédrale $»^{46}$, ceux de la rationalité orthogonale et ceux en quête douce de transcendance et d'absolu, puissent communiquer dans un langage universel de l'humanisme?

\title{
THE PRESIDENT'S PHANTOM GESTURE: \\ RECONFIGURATIONS BETWEEN A SECULAR STATE AND RELIGION IN TIMES OF POST-SECULARISM
}

\begin{abstract}
Summary
The personal attitude of the successive presidents of the Fifth Republic of France to religion, although different, did not violate the general principle of the secularity of the state. Meanwhile, the ambiguity of some gestures of president Emmanuel Macron, visible during the funeral ceremony of Johnny Hallyday, sparked protests among the laicity defenders, especially since it fell exactly on the anniversary of the 1905 law on the separation of Churches from the State. Is it possible to see in the president's behaviour signs of striving to push the limits of secularity that seemed so far unshakeable? An analysis of Macron's speeches, forming a kind of political theology, will allow us to feature the outlines of the (new) post-secular laicity that is emerging during his presidency.
\end{abstract}

Key words: post-secularism, laicity, Macron, Fifth Republic, religion.

45 J. Habermas, Entre naturalisme et religion. Les défis de la démocratie, Gallimard, Paris 2008.

${ }^{46}$ G. Weigel, The Cube and the Cathedral Europa. America and Politics without God, Basic Books, New York 2005. 